those individuals aged $\geq 75$ years, there was a considerable rise in the proportion of patients without hypertension who had an antithrombotic bleed. This finding corresponds with the marked difference in antithrombotic use between OCSP and OXVASC (4\% vs $41 \%$ ).

The authors conclude that, while efforts to treat hypertension have reduced the incidence of $\mathrm{IH}$ in individuals under 75 years of age, they have had little effect in older individuals, in whom antithrombotic use seems to be a more important risk factor. Given the aging population in the UK, the absolute number of cases of IH might increase if the risk associated with antithrombotic use is not addressed.

Original article Lovelock CE et al. (2007) Change in incidence and aetiology of intracerebral haemorrhage in Oxfordshire, UK, between 1981 and 2006: a populationbased study. Lancet Neurol 6: 487-493

\section{Myocardial injury predicts poor outcome in patients with acute liver failure}

Elevated troponin I levels, indicative of cardiac muscle damage, are found in some patients with sepsis or acute stroke. Heart problems sometimes occur in patients with acute liver failure (ALF), but whether cardiac damage is a common occurrence in these patients is unknown. To shed some light on the matter, Parekh et al. investigated the prevalence of elevated troponin I levels in patients with ALF for the Acute Liver Failure Study Group.

The study population comprised 187 patients from the US ALF registry. Serum troponin I levels indicative of myocardial injury (defined as a troponin I level $>0.1 \mathrm{ng} / \mathrm{ml}$ ) were found in 138 participants (74\%). Increasing troponin I levels correlated with advanced hepatic coma, death, and death after transplant. For those with elevated troponin I levels, the odds ratios for advanced coma and death were 3.88 and 4.69 , respectively. Three weeks after enrollment, $34.4 \%$ of those with elevated troponin I levels had died, compared with $10.2 \%$ of those with normal levels. Troponin I levels also correlated positively with APACHE II scores, and negatively with glomerular filtration rate.

The author conclude that, although the mechanism of cardiac injury in patients with ALF remains unknown, subclinical cardiac damage could have a considerable negative impact on the outcome for patients with ALF. Troponin I might be useful both as a marker of cardiac injury and as a predictor of outcome.

Original article Parekh NK et al. (2007) Elevated troponin I levels in acute liver failure: is myocardial injury an integral part of acute liver failure? Hepatology 45: 1489-1495

\section{Trimetazidine reduces risk of contrast-induced nephropathy after coronary procedures}

Contrast-induced nephropathy (CIN), a serious complication of coronary angiography, is particularly common in patients with renal insufficiency. Hydration with isotonic saline is the only generally accepted way of preventing $\mathrm{CIN}$ at present. As formation of oxygen free radicals and renal medullar ischemia are thought to be involved in the pathogenesis of CIN, Onbasili et al. investigated whether administration of trimetazidine, an agent with anti-ischemic and antioxidant properties, might be a useful adjunct to isotonic saline.

The study enrolled 82 patients with mild renal insufficiency who underwent coronary procedures. Patients were randomly assigned to a trimetazidine group (20 mg thrice daily orally for $72 \mathrm{~h}$ starting $48 \mathrm{~h}$ before the procedure; $n=40)$ or a control group $(n=42)$. All patients received standard parenteral hydration (isotonic saline $1 \mathrm{ml} / \mathrm{kg} / \mathrm{h}$ from $12 \mathrm{~h}$ before the procedure to up to $12 \mathrm{~h}$ after). The same contrast agent-iopromide-was used in all patients.

CIN (defined as an absolute serum creatinine increase of $44 \mu \mathrm{mol} / \mathrm{l}[0.5 \mathrm{mg} / \mathrm{dl}]$ or a relative increase of $25 \%$ from baseline 1-2 days after the procedure) was significantly more common in controls than in trimetazidine-treated patients (16.6\% vs $2.5 \% ; P<0.05)$. In controls, serum creatinine levels were higher and creatinine clearances were lower 2 days after the procedure than at baseline; these parameters returned to baseline levels by day 7. By contrast, no changes from baseline in serum creatinine levels or creatinine clearances were seen on day 2 in trimetazidine-treated patients, and by day 7 these patients actually had decreased serum creatinine levels and increased creatinine clearances. No trimetazadine-related adverse effects were noted.

Original article Onbasili AO et al. (2007) Trimetazidine in the prevention of contrast-induced nephropathy after coronary procedures. Heart 93: 698-702 